\title{
Duplex Bioelectronic Tongue for Sensing Umami and Sweet Tastes Based on Human Taste Receptor Nanovesicles
}

\author{
Sae Ryun Ahn, ${ }^{\dagger, \mathbb{I}}$ Ji Hyun An, ${ }^{\ddagger, \mathbb{L}}$ Hyun Seok Song, ${ }^{\perp}$ Jin Wook Park, ${ }^{\ddagger}$ Sang Hun Lee, ${ }^{\S}$ Jae Hyun Kim," \\ Jyongsik Jang, ${ }^{*}+$ and Tai Hyun Park ${ }^{*} \dagger, \nabla$ \\ ${ }^{\dagger}$ School of Chemical and Biological Engineering, Bio-MAX Institute, Seoul National University, Seoul 151-744, Republic of Korea \\ ${ }^{\ddagger}$ School of Chemical and Biological Engineering, Seoul National University, Seoul 151-744, Republic of Korea \\ ${ }^{\perp}$ Division of Bioconvergence Analysis, Korea Basic Science Institute (KBSI), Yuseong, Daejeon 169-148, Republic of Korea \\ ${ }^{\S}$ Department of Bioengineering, University of California, Berkeley, California 94720, United States \\ "S.LSI Material Technology Group, Device Solutions, Samsung Electronics, 1, Samsung-ro, Giheung-gu, Yongin-si, Gyeonggi-do \\ 446-711, Korea \\ ${ }^{\nabla}$ Advanced Institutes of Convergence Technology, Suwon, Gyeonggi-do 443-270, Republic of Korea
}

\section{Supporting Information}

ABSTRACT: For several decades, significant efforts have been made in developing artificial taste sensors to recognize the five basic tastes. So far, the well-established taste sensor is an E-tongue, which is constructed with polymer and lipid membranes. However, the previous artificial taste sensors have limitations in various food, beverage, and cosmetic industries because of their failure to mimic human taste reception. There are many interactions between tastants. Therefore, detecting the interactions in a multiplexing
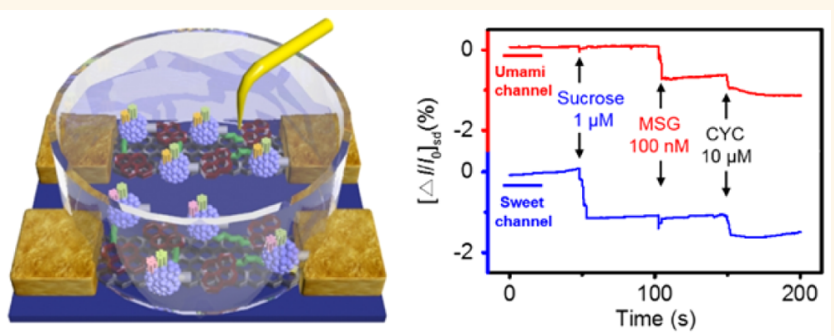
system is required. Herein, we developed a duplex bioelectronic tongue (DBT) based on graphene field-effect transistors that were functionalized with heterodimeric human umami taste and sweet taste receptor nanovesicles. Two types of nanovesicles, which have human T1R1/T1R3 for the umami taste and human T1R2/T1R3 for the sweet taste on their membranes, immobilized on micropatterned graphene surfaces were used for the simultaneous detection of the umami and sweet tastants. The DBT platform led to highly sensitive and selective recognition of target tastants at low concentrations (ca. $100 \mathrm{nM}$ ). Moreover, our DBT was able to detect the enhancing effect of taste enhancers as in a human taste sensory system. This technique can be a useful tool for the detection of tastes instead of sensory evaluation and development of new artificial tastants in the food and beverage industry.

KEYWORDS: G-protein coupled receptor (GPCR), taste receptor, nanovesicle, bioelectronic tongue, duplex taste sensor, graphene, field-effect transistor (FET)

r he sense of taste provides humans with various information about food and environments. ${ }^{1}$ Humans can recognize five basic tastes: sweet, umami, bitter, salty, and sour. ${ }^{2}$ Among the five tastes, the umami and sweet tastes are attractive to humans. The fascination with the two tastes is due to not only their pleasant flavors but also the need for their nutritional values in the bodies. Umami represents the taste of L-amino acids, which have functions as the unit of protein blocks, biosynthetic precursor, and metabolic fuels. ${ }^{3}$ Moreover, the sweet taste includes glucose and various forms of sugar, which are essential components for energy production. Over the past decade, detection tools of the two tastes have been developed using molecular biology and electrochemis- try. ${ }^{4-14}$ Recently, artificial taste sensors are very well developed as electronic tongues. These are constructed with inorganic materials and lipid membranes as sensor arrays. ${ }^{12-14}$ However, these electronic tongues are not selective enough to mimic humans' taste system. In the case of human sweet taste receptor, the ligand sweeteners are not only small-molecular weight sweeteners such as sucrose but also large-molecular weight sweet proteins. ${ }^{15}$ Umami taste ligand molecules include glutamates and nucleotides. Furthermore, they cannot test for

Received: April 15, 2016

Accepted: June 21, 2016

Published: June 21, 2016 


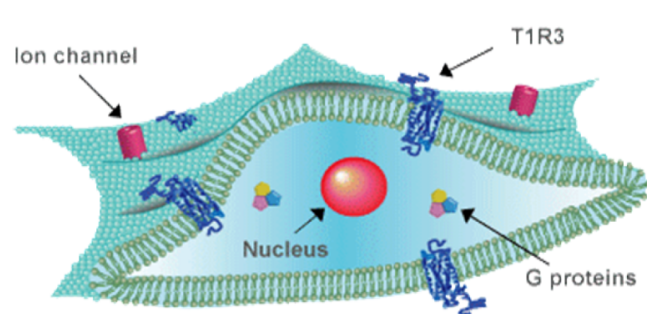

HEK-293 cell stably expressing T1R3

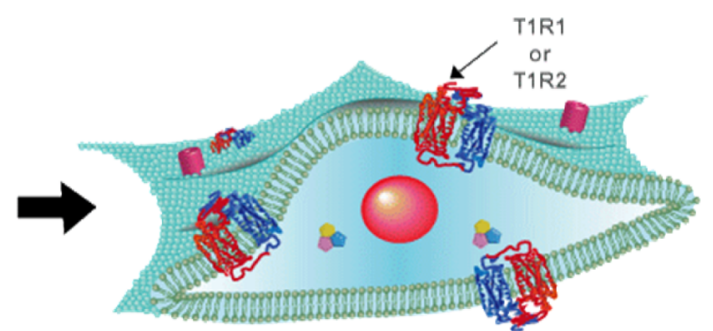

Expression of T1R1 of T1R2

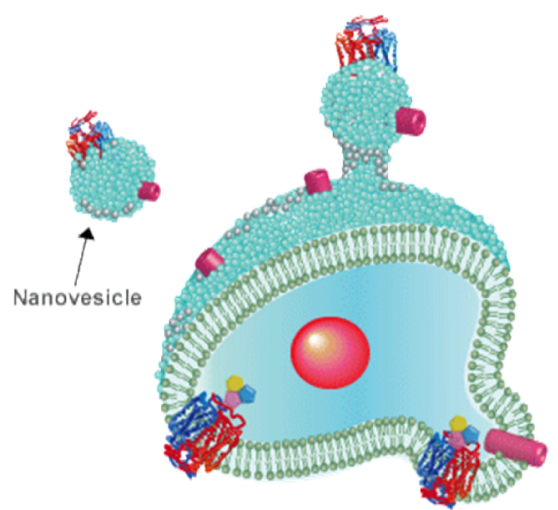

Formation of nanovesicles via agitation with cytochalasine $B$
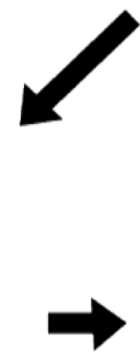

Structure of a nanovesicle

Figure 1. Schematic illustration representing the construction of nanovesicles expressing heterodimeric human taste receptors. T1R3 was stably expressed in HEK-293 cells, and the cells were transfected with either T1R1 or T1R2. This resulted in the expression of heterodimeric taste receptors, T1R1/T1R3 (umami taste receptor) and T1R2/T1R3 (sweet taste receptor). The constructed nanovesicles have taste receptors and ion-channels on their membrane.

unknown compounds, because they are established after screening chemical and electrical properties of every tastant one by one. There are many unknown compounds that have tastes in foods. For understanding their interactions with diverse tastants, other approaches are needed.

In our previous research, the use of human G-protein coupled receptors (GPCRs) was suggested for the development of a human mimic sensor. ${ }^{16,17}$ Multicellular organisms were evaluated by communication with their environment using membrane-bound receptors. Among these membrane-bound receptors, GPCRs are most diverse, ${ }^{18}$ because they play an important role in recognizing and transducing messages. Therefore, the high-throughput screening of the GPCRs was extensively studied. ${ }^{19}$ Despite the necessity of most of the GPCRs to form monomers, the two taste receptors consist of the complexes of two heteromeric members of the T1R family of GPCRs: T1R1/T1R3, an umami taste receptor, and T1R2/ T1R3, a sweet taste receptor., ${ }^{2,7,20,21}$ Our previous human tongue-like nanovesicle-based bioelectronic tongue using heterodimeric human sweet taste receptor nanovesicles and swCNT-FETs successfully discriminated sweeteners. The sweetener sensor enabled a biosensor with human-like broad selectivity for the detection of sweeteners. ${ }^{17}$

Herein, we developed a duplex bioelectronic tongue (DBT) using graphene field-effect transistors (FETs) that were conjugated to the human taste receptor nanovesicles. The combination of heterodimeric taste receptors, the umami and sweet taste receptors, facilitated cellular signal transduction by taste perception via the nanovesicle. Our DBT platform utilizing heterodimeric taste receptors in human displayed the capability to detect various tastants such as many types of umami and sweet molecules with high sensitivity and selectivity. The graphene transducers provided excellent sensing properties such as extraordinary sensitivity, stable operation, and rapid response. The DBT was able to detect both umami and sweet tastes simultaneously and also detect the taste enhancing effect of taste enhancers. In addition, the sensing capability of our platform was demonstrated in both the mixture of tastes and the panel of food samples.

\section{RESULTS AND DISCUSSION}

Expression of Heterodimeric Human Taste Receptors and Construction of Nanovesicles. Figure 1 shows the schematic illustration representing the production of nanovesicles expressing heterodimeric human taste receptors. A human TAS1R3 inserted in a mammalian expression vector was expressed in human embryonic kidney-293 (HEK-293) cells, and cells stably expressing T1R3 were generated through antibiotic selection. Then, T1R1 or T1R2 was transiently expressed in the T1R3-expressing HEK-293 cell-lines. As a result, the human umami taste receptor was composed of T1R1 and T1R3, and the sweet taste receptor was composed of T1R2 and T1R3. Stable T1R3 expression was confirmed from the membrane fractions of HEK-293 cells by Western blot analysis using an anti-flag antibody against flag-tag fused with T1R3 (Figure 2a). Transient expression of T1R1 and T1R2 in the umami and sweet taste receptor cells were confirmed by Western blot analysis using anti-T1R1 and anti-T1R2 antibodies. To probe into the intracellular signal transduction in the taste receptor cells, calcium ion influx was measured upon the addition of their tastants by the calcium signal assay (Figure 2c). While the taste receptor cells expressing heterodimeric 
a b

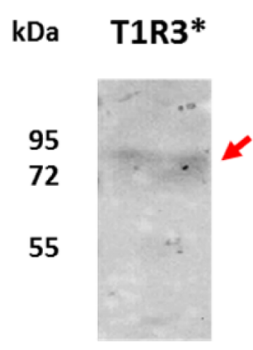

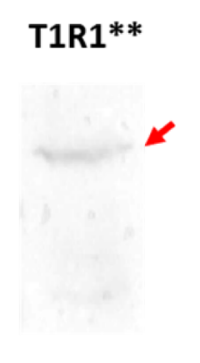

T1R2**

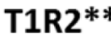

C (1)

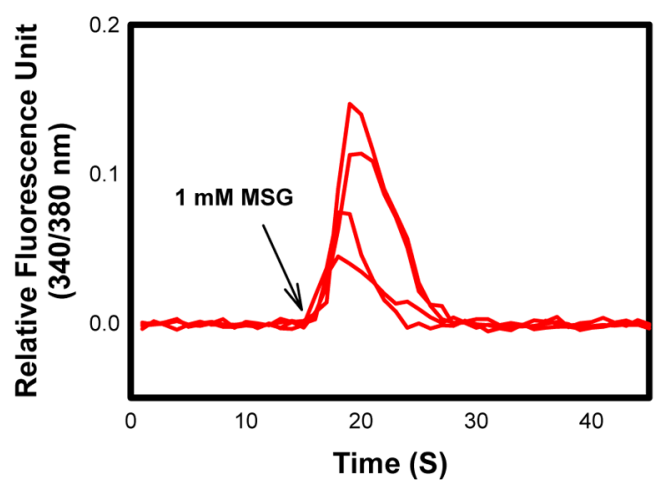

d (1)

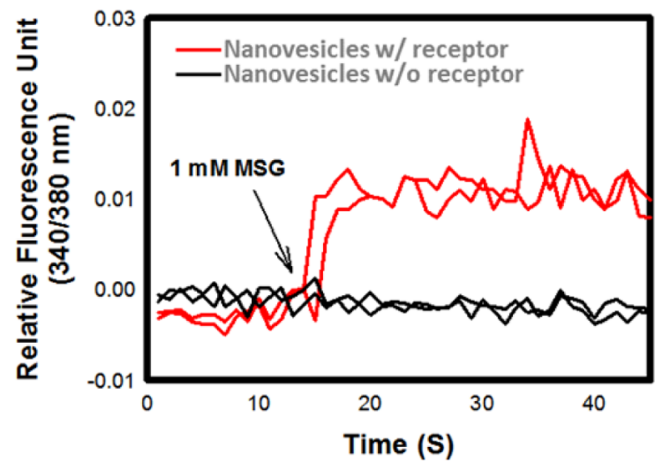

(2)

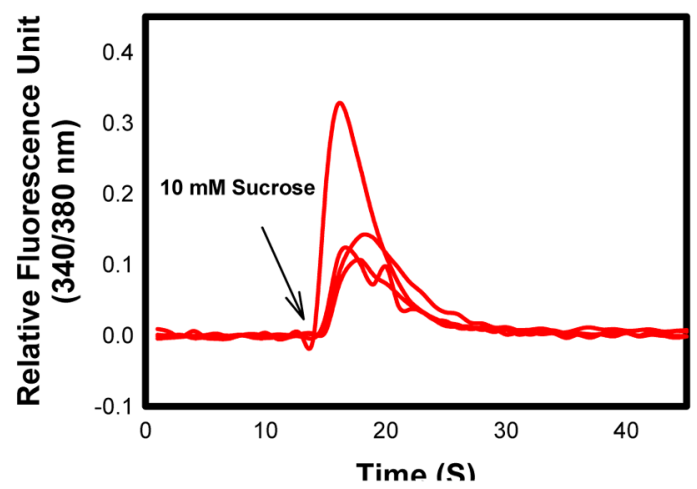

(2)

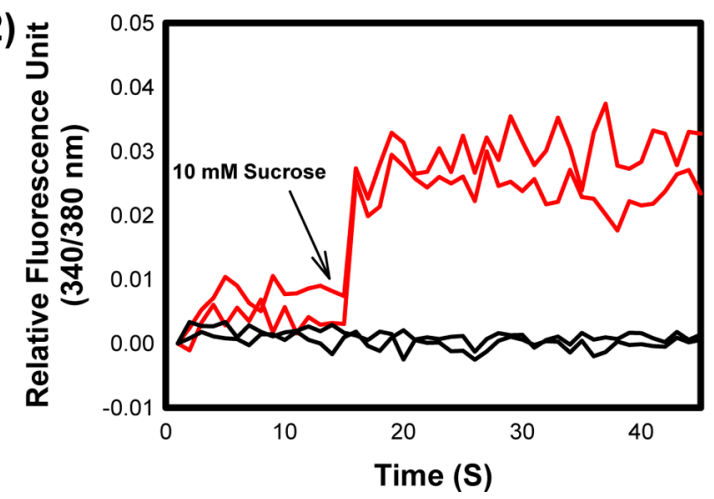

Figure 2. Confirmation of expression and signal transduction activity. (a) Western blot analysis of membrane fractions of HEK-293 cells transfected with umami and sweet taste receptors: *stable cell line with T1R3; **transient expression in T1R3 stable cell line. (b) Western blot analysis of T1R1, T1R2, and T1R3 in the nanovesicles: *stable cell line with T1R3, **transient expression in T1R3 stable cell line. (c) $\mathrm{Ca}^{2+}$ signal analysis using (1)umami taste receptor cells, T1R1/T1R3-expressed, upon the addition of $1 \mathrm{mM}$ of MSG and (2) sweet taste receptor cells, T1R2/T1R3-expressed, upon the addition of $10 \mathrm{mM}$ of sucrose. (d) $\mathrm{Ca}^{2+}$ signal analysis using (1) umami taste receptor nanovesicles upon addition of $1 \mathrm{mM}$ of MSG and (2) sweet taste receptor nanovesicles upon addition of $10 \mathrm{mM}$ of sucrose.

receptors show signals, monomeric receptor expressing cells have no signals upon the addition of the tastants (Figure S1 in the Supporting Information). This result shows that human umami and sweet taste receptors exist and activate only in the form of heterodimeric receptors. ${ }^{22}$ The relatively smaller signal in F340/F380 change may be caused by the lower expression level for heterodimeric receptors than other monomeric GPCRs. It would mimic the human taste system more closely if the other downstream transduction machineries such as $\mathrm{G} \alpha$ gustducin, PLC $\beta 2$, and TRPM5 were additionally transfected.

Then, the taste receptor expressing cells were gently agitated after treatment with cytochalasin B, which destroys the cytoskeleton to make the cell membrane unstable. Nanovesicles with taste receptors on their membranes were budded off from the taste receptor-expressing HEK-293 cells. The constructed nanovesicles have intracellular signaling machineries such as taste receptors and ion channels on their membrane. It is likely that adding additional downstream transduction elements may improve the signal change. The expression of each receptor on the nanovesicles was confirmed by Western blot analysis using anti-flag tag antibody for T1R3 and anti-T1R1 and anti-T1R2 antibodies for T1R1 and T1R2, respectively (Figure 2b). To investigate whether the specific tastants to each taste receptor induce intracellular transduction in the nanovesicles, calcium influx test was carried out (Figure 2d). These results indicate that the nanovesicles reserve their original function, which is the signal transduction of the taste receptor cells. In our previous work, we measured the response to the sweet tastant in the solution without $\mathrm{Ca}^{2+}$, and no signal change was observed. ${ }^{17}$ This shows that the signal change is due to the calcium influx from the outside of the cell or nanovesicle. However, the calcium signal was not fully restored to the baseline. The probable reason is that the nanovesicles cannot pump out the calcium ions, because of the lack of ion pumps and calmodulins. ${ }^{17,23,24}$

Fabrication of Duplex Bioelectronic Tongue. Transducers play a crucial role in improving the performance of biosensors. Graphene is one of the high-performance materials 


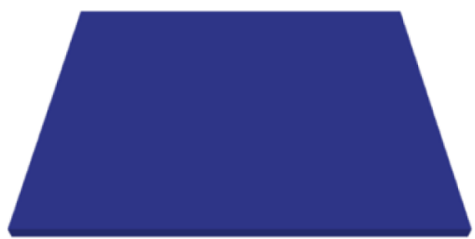

$\mathrm{SiO}_{2}$ substrate

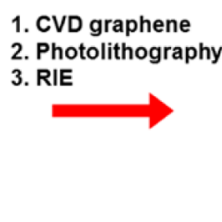

2. Photolithography

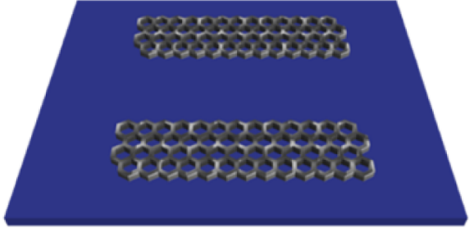

Patterned graphene substrate

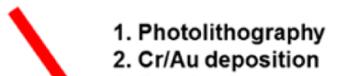

2. Cr/Au deposition

3. Passivation

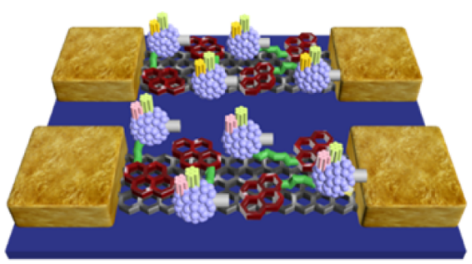

DGE/PSE/Nanovesicles

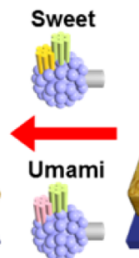

Nanovesicle

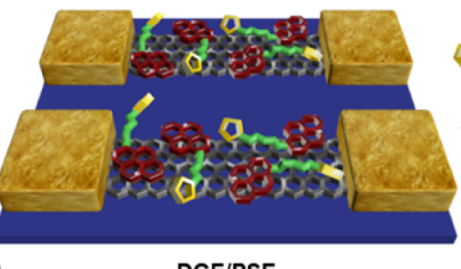

DGE/PSE

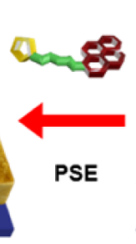

E

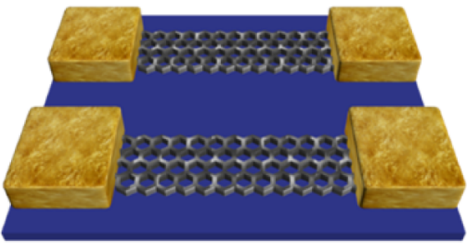

Duplex graphene electrode (DGE)

Figure 3. Schematic illustrations for the fabrication procedure of duplex sensor containing umami and sweet taste receptor nanovesicles.

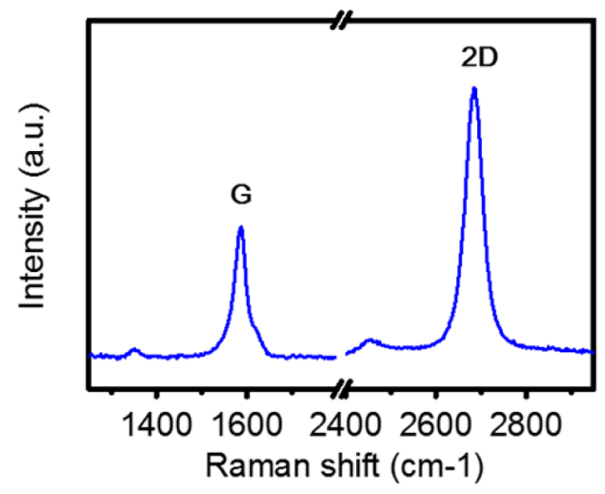

b

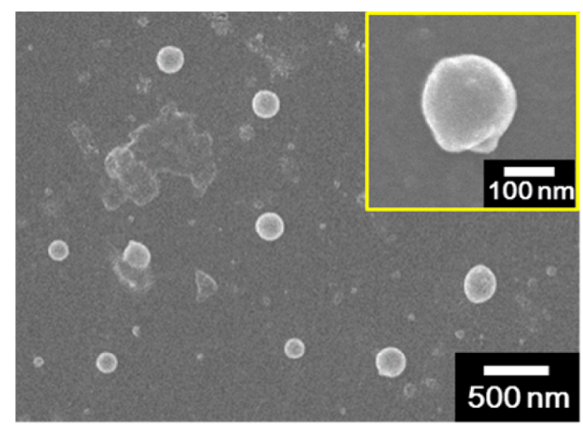

C

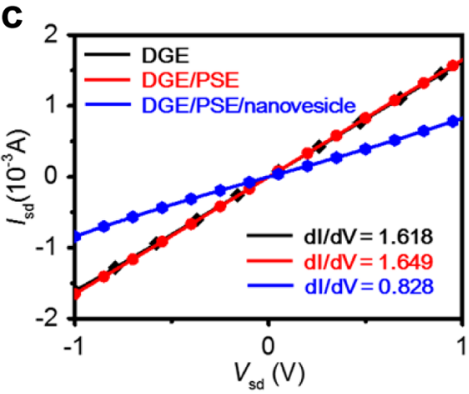

d

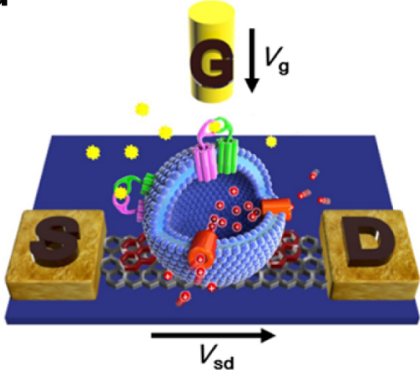

e

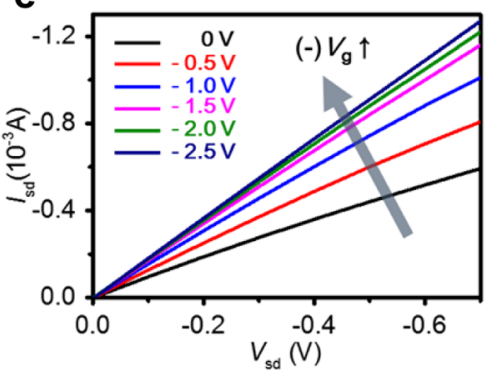

Figure 4. Characterization and electrical property of DBT sensors. (a) Raman spectra of CVD graphene on a silicon oxide substrate. (b) FESEM image of a GM channel after the introduction of nanovesicles. (c) Current-voltage $(I-V)$ curves of the DBT sensor before and after the immobilization of nanovesicles. (d) Schematic diagram depicting the FET geometry and showing the sensing mechanism of a DBT. (e) Output characteristics of a FET-type DBT $\left(V_{g}, 0\right.$ to $-2.5 \mathrm{~V}$ in a step of $-0.5 \mathrm{~V}$, and $V_{\text {sd }} 0$ to $-0.7 \mathrm{~V}$ in a step of $\left.-50 \mathrm{mV}\right)$.

for sensor transducers. It has a large surface area, high conductivity, and superb carrier mobility, ${ }^{25,26}$ providing outstanding sensing properties for biosensors. ${ }^{27,28}$ To take the advantage of this special aspect, we fabricated FET-type taste sensors with chemical vapor deposition (CVD)-grown graphene. Figure 3 presents the schematic illustration for the duplex bioelectronic tongue (DBT) based on taste receptor nanovesicles. Graphene-FETs for DBTs were prepared by the method reported previously. ${ }^{29}$ Single layer graphene was fabricated by the CVD process. Subsequently, the graphene was positioned on a four-inch silicon oxide wafer using the dry- transfer method. Photolithography and reactive-ion etching (RIE) processes were used to prepare graphene micropatterns (GMs). Afterward, a second photolithography process was conducted to provide the photoresist patterns for contact electrodes that were utilized for the source (S) and drain (D) electrodes. The contact electrodes were deposited on the GM channels by the thermal evaporation of metals $(\mathrm{Cr} / \mathrm{Au})$ and liftoff method. Finally, dual-channel graphene based FETs for DBTs were constructed after the passivation process.

To introduce the nanovesicles on the GM channels, the surface of the GMs was exposed to pyrenebutyric acid $\mathrm{N}$ - 


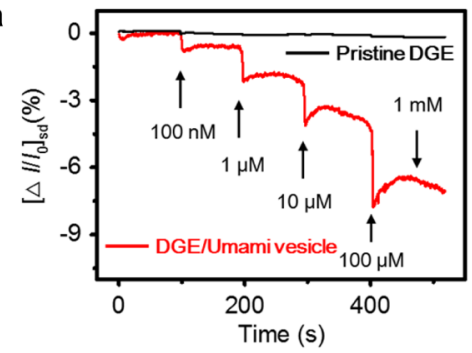

C
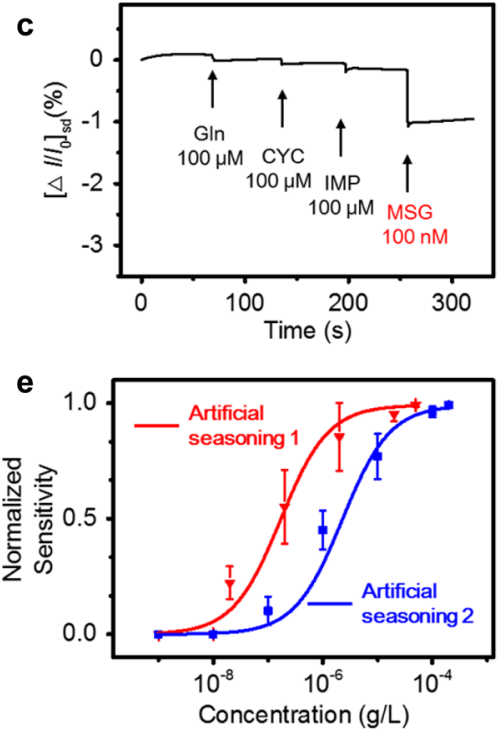

b

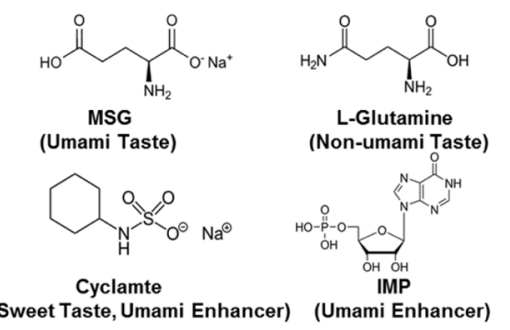

d

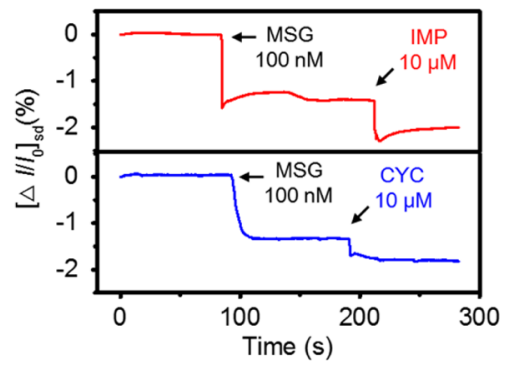

Figure 5. Discrimination of umami tastants with umami taste nanovesicle-immobilized bioelectronic tongues. (a) Real-time response of a single channel in a DBT with various concentrations of MSG (100 nM to $1 \mathrm{mM}$ ). DGE stands for duplex graphene electrode. (b) Chemical structures for sensing tests related to umami taste. (c) Selective response of a DBT toward target tastant (MSG, $100 \mathrm{nM}$ ). (d) Real-time electrical measurements of DBTs toward umami enhancers (IMP and CYC, $10 \mu \mathrm{M}$ ). (e) Dose-dependent responses of DBTs to artificial seasonings.

hydroxysuccinimide ester (PSE) as the linker molecules. The PSE was placed on the GM channels via $\pi-\pi$ stacking. The pyrenyl groups of the PSE were bound to the plane of the graphene. ${ }^{30,31}$ Then, the PSE-functionalized GMs were treated with the nanovesicles containing umami or sweet taste receptors. The succinimidyl groups of the PSE interacted with the proteins of the vesicles, resulting in chemically stable peptide bonding. Therefore, an immobilized nanovesicle sensor system was successfully fabricated on a silicon oxide substrate. Two GM channels of a DBT were used for the simultaneous detection of umami and sweet tastants. One channel was connected to umami vesicles, and the other channel was treated with sweet vesicles. As a result, the DBT sensor could simultaneously detect dual responses for umami and sweet tastants.

Characterization of Duplex Bioelectronic Tongue. A fabricated multiplexed graphene electrode (MGE) circuit is represented in the photograph (Figure S2 in Supporting Information). The MGE consists of eight pairs of source (S) and drain (D) electrodes, and two pairs of the contact electrodes were utilized for our DBT configuration. CVDgrown graphene was used as the electrical channel between contact electrodes. The number of layers and thickness of the graphene was measured by Raman spectra and high-resolution transmission electron microscopy (HR-TEM) (Figure 4a). Raman analysis was conducted to measure the distinctive peaks of the graphene. In a single layer graphene, the $2 \mathrm{D}$ peak has stronger and sharper intensity than the $G$ peak, which distinguishes it from double and few layer graphene. ${ }^{32}$ As shown in Figure 4a, the G peak $\left(\sim 1590 \mathrm{~cm}^{-1}\right)$ and $2 \mathrm{D}$ peak $\left(\sim 2690 \mathrm{~cm}^{-1}\right)$ were consistent with single layer graphene. In order to further clarify the number of layers, the edge of the graphene was analyzed using HR-TEM, which is a powerful tool to visualize the graphene layer. The HR-TEM image displays the presence of single layer graphene (Figure S2 in Supporting Information).

Nanovesicles immobilized on a graphene channel were observed by field-emission scanning electron microscopy (FESEM), as shown in Figure $4 \mathrm{~b}$. The lyophilization process was performed to preserve the shape of the nanovesicles for the FESEM analysis. Figure $4 \mathrm{~b}$ clearly shows that the nanovesicles were successfully attached to the surface of the graphene. As shown in the SEM image, the nanovesicles showed spherical shape with diameters in the range 100-200 nm.

To exploit the electrical characteristics of the DBT configuration, current-voltage $(I-V)$ measurement was carried out (Figure 4c). The graph in Figure 4c exhibits the variations in the $I-V$ values before and after nanovesicle immobilization on the surface of the GM channel. The $I-V$ relation maintained linear properties, even though the $\mathrm{d} I / \mathrm{d} V$ value moderately decreased after nanovesicle attachment. It was apparent that stable ohmic contact remained during the immobilization process. These results demonstrate that the DBT transducers can preserve a reliable electrical contact, indicating that an interaction between target tastant and nanovesicles can be detected by observing the changes in the current. 


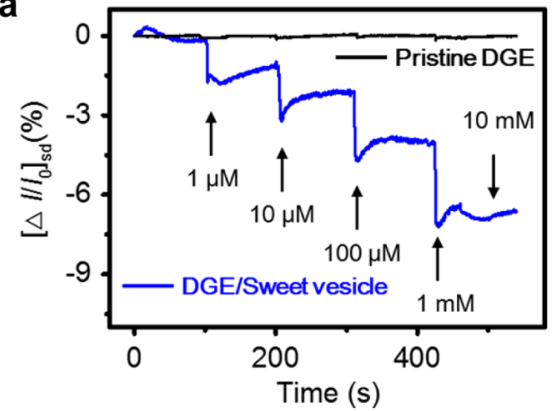

C

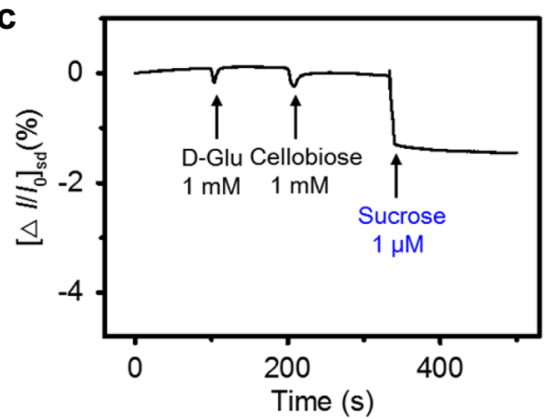

b

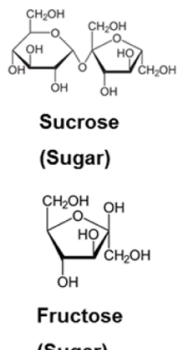

(Sugar)
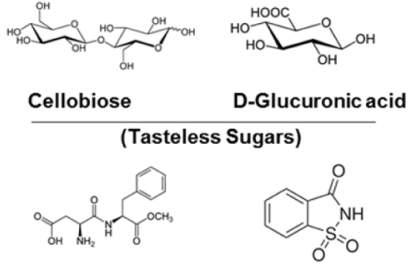

Aspartam Saccharin

(Artificial Sweeteners)

d

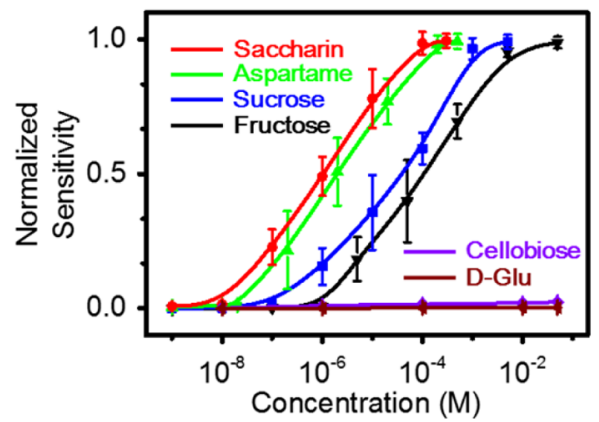

Figure 6. Sensing performance of sweet taste receptor nanovesicle-based bioelectronics tongues. (a) Real-time electrical measurement of a DBT to various sucrose concentrations $(1 \mu \mathrm{M}$ to $10 \mathrm{mM})$. DGE stands for duplex graphene electrode. (b) Chemical structures of diverse sweet tastants. (c) Selective property of a DBT toward target tastant (sucrose, $1 \mu \mathrm{M}$ ) and nontarget tastants (cellobiose and D-glucuronic acid, $1 \mathrm{mM})$. (d) The normalized sensitivity $\left(\Delta I / \Delta I_{\max }\right)$ of DBTs toward tasteless natural and artificial sweet tastants.

Exploring the possibility to utilize nanovesicle-immobilized GM channels as transducers in the FET system, a liquid-ion gated FET geometry was fabricated. Figure $4 \mathrm{~d}$ illustrates the setup of the FET system used to investigate the electrical characteristics under different gate voltages $\left(V_{\mathrm{g}}\right)$. Dulbecco's phosphate buffered saline (DPBS; $\mathrm{pH}$ 7.4) was used as the electrolyte. A gate electrode was placed in the DPBS electrolyte, providing that the transducer has a reliable contact with the solution. Figure $4 \mathrm{e}$ reveals the output curves of the DBT under various $V_{\mathrm{g}}$ ranging from 0 to $-3 \mathrm{~V}$. The drain-to-source current $\left(I_{\mathrm{sd}}\right)$ negatively increased according to negatively increasing $V_{\mathrm{g}}$, meaning p-type semiconductor behavior (hole-transporting). Moreover, the ohmic contact remained at the different $V_{\mathrm{g}}$, suggesting that the sensing signal changes were mainly affected by the electrostatic gating effect of the GM channel, rather than the contact resistance. Considering these results, the DBT can transduce the sensing signal created when charge-carrier density is changed by the interaction between the nanovesicles and specific-target molecules. Thus, the liquid-ion gated FET system facilitates a label-free detection of taste molecules.

Real-Time Responses of the DBT for Umami Tastant. In our previous investigation, graphene-based FET sensors demonstrated stable and responsive performance in the p-type region because of the adsorption of oxygen, whereas the graphene-based sensors had ambipolar properties. ${ }^{33}$ Therefore, real-time responses in this study were measured in the p-type region.

Figure $4 \mathrm{~d}$ illustrates the sensing mechanism of the grapheneFET type DBT platform. First, the specific binding between the taste receptor and target tastant-induced signal transduction inside the nanovesicles via G-proteins and ion channels introduced $\mathrm{Ca}^{2+}$ ions into the nanovesicles. Subsequently, the accumulated ions applied a positive gate effect on the surface of the p-type sensors. Finally, the positive potential effect reduced the number of holes in the graphene and thus decreased the $I_{\text {sd }}$.

As shown in Figure 5a, the real-time responses of the DBT device were observed by monitoring the $I_{\mathrm{sd}}$, after adding various monosodium glutamate (MSG) concentrations. The $I_{\text {sd }}$ value immediately decreased upon exposure to MSG as umami tastant, coinciding with the sensing mechanism of the DBT device. The minimum detectable level (MDL) was $\sim 100 \mathrm{nM}$ (signal-to-noise ratio 3.10), and the saturation level was $\sim 1$ $\mathrm{mM}$. The detection limit $(100 \mathrm{nM})$ of the DBT was 1000 -times more sensitive than those of other taste sensors using polymer membranes and lipid membranes. ${ }^{11-14}$ The current changes were detected over a wide range of MSG concentrations $\left(10^{-7}\right.$ to $10^{-3} \mathrm{M}$ ), and the current signals gradually decreased in response to higher concentrations of MSG. However, the pristine duplex graphene electrode (DGE) in the absence of the nanovesicle did not show any critical changes in the $I_{\mathrm{sd}}$ after the introduction of MSG, indicating that the specific interaction between the nanovesicles and umami tastant triggered prompt current changes. Moreover, the DBT exhibited a highly rapid response (on a time scale of less than $1 \mathrm{~s}$ ).

Figure $5 b$ displays the chemical structures of MSG, Lglutamine (nonumami taste), and umami enhancers. The chemicals were used to prove the specificity of our DBT device, as shown in Figure 5c. Although the nontarget chemicals had much higher concentrations (3 orders of magnitude) than target MSG, no significant changes occurred following the injection of the nontarget chemicals. However, the addition of $100 \mathrm{nM}$ concentration of MSG remarkably affected the current changes in $I_{\text {sd }}$ indicating that the DBT features the ability to perceive the target tastant with superior selectivity.

Figure $5 \mathrm{~d}$ shows the enhancing effect of the taste enhancers. Inosine-5'-monophosphate (IMP) and cyclamate (CYC) are known as umami taste enhancers. ${ }^{34,35}$ While IMP and CYC did 


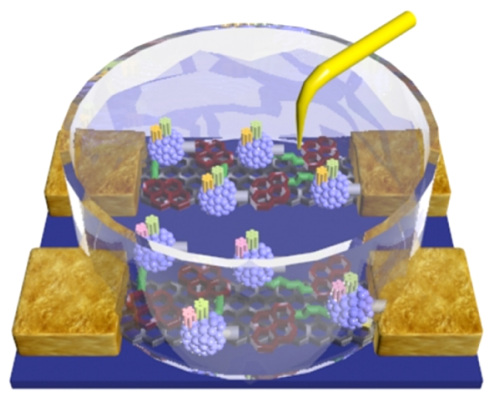

b
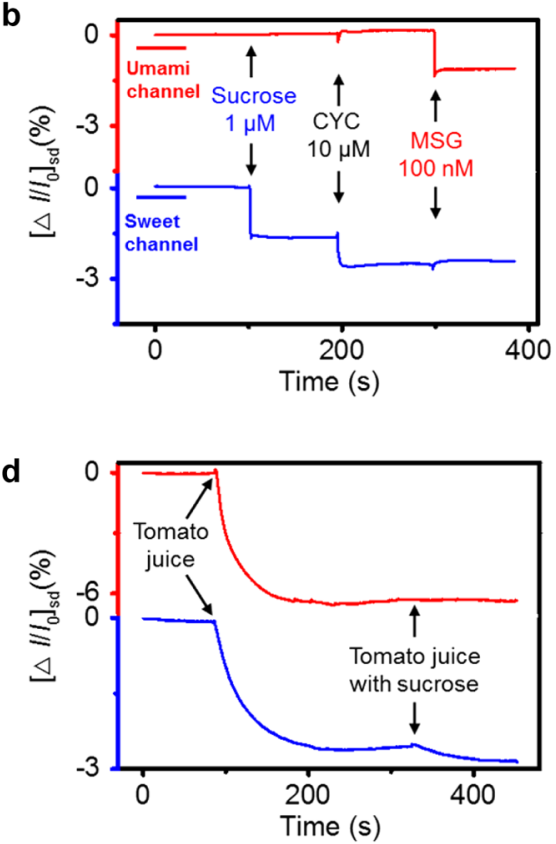

C

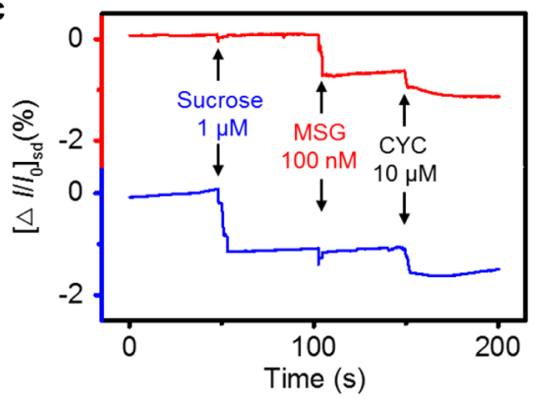

e

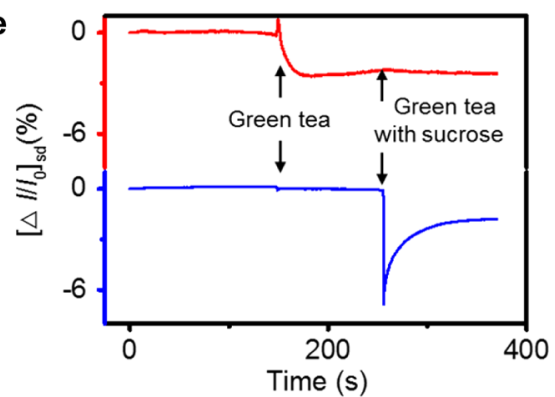

Figure 7. Simultaneous detection of umami and sweet tastants using DBTs. (a) Schematic diagram of DBT geometry. (b) Real-time response of two channels in the DBT sensor toward umami and sweet tastants. (c) Real-time electrical measurement of a DBT for monitoring the enhancing property of CYC. Real-time responses from (d) tomato juice solution and (e) green tea solution as real drink samples (red and blue lines indicate umami and sweet channels, respectively).

not show any significant change when the enhancers were introduced alone in the DBT system (Figure 5c), the enhancers affected the responses in the presence of MSG. As predicted, the DBTs responsed to the addition of MSG (100 nM) and were stimulated by the introduction of the respective IMP or CYC $(10 \mu \mathrm{M})$. These results demonstrate that the responses of umami receptors are significantly magnified by the synergistic effect, the combined stimulation of MSG and umami enhancers.

Normalized sensitivity $\left(\Delta I / \Delta I_{\max }\right)$ presents dose-dependent responses of the DBT platform to commercially available artificial seasonings, as shown in Figure 5e. Artificial seasonings are often used for the umami taste. ${ }^{36}$ L-Glutamic acid, a wellknown umami tastant, is contained in various foods such as kelp, mushrooms, and fish sauce. However, it is difficult to sense the umami taste in those foods because of the low solubility of the glutamic acid. ${ }^{37-39}$ Therefore, artificial seasonings containing MSG, which is soluble glutamic acid, are normally used in food. The artificial seasoning 1 contains salt, sugars (sucrose, lactose), flavor raw material (dried bonito powder, bonito extract), yeast extract, wheat protein-fermented seasoning, and yeast extract-fermented seasoning. The artificial seasoning 2 contains 97.3\% MSG, 1.35\% IMP, and 1.35\% guanosine monophosphate (GMP). The concentration of artificial seasonings commonly used in food is in the range 1-10 g/L. However, in these experiments, much lower concentrations of the artificial seasonings were detected in the range $10^{-8}-10^{-4} \mathrm{~g} / \mathrm{L}$, indicating that the sensitivity of DBT is sufficient to be applied in the food industry.

Real-Time Responses of the DBT for Sweet Tastant. Figure 6a displays the real-time responses of the sweet taste sensor. The $I_{\mathrm{sd}}$ of the sensor decreased because of the interaction between the sweet taste receptor nanovesicles and sucrose and was entirely consistent with the sensing mechanism of the previous umami sensor. The current changes were observed within $1 \mathrm{~s}$ and detected over an extensive range of sucrose concentrations ( $1 \mu \mathrm{M}$ to $10 \mathrm{mM})$, whereas a sensor without nanovesicle as a control experiment exhibited only slight variation. The DBT of the sucrose sensor was $\sim 1 \mu \mathrm{M}$ (signal-to-noise ratio 5.38), and the saturating concentration was $\sim 10 \mathrm{mM}$. The limit of detection (LOD) in the DBT was 1 $\mu \mathrm{M}$ and was 100-times more sensitive than that of our previously reported sensor based on a swCNT-FET. ${ }^{17}$ The outstanding properties of CVD graphene contributed to this enhanced performance. The $2 \mathrm{D}$ graphene channel had a larger area than 1D CNT channels, enabling more nanovesicles to be loaded in the channel of the graphene-FET sensor. Moreover, 
the graphene featured high conductivity and fast carrier mobility, leading to improved signal transduction.

To further characterize the sensing performance of the DBT for the sweet taste, selectivity experiments were conducted. Figure $6 \mathrm{~b}$ presents the chemical structures of natural sugars (sucrose and fructose), tasteless sugars, and artificial sweeteners for the sensing experiments. The selective response of the DBT to sucrose is shown in Figure 6c. The $I_{\text {sd }}$ values changed significantly when sucrose $(1 \mu \mathrm{M})$ as the target sugar was introduced in the DBT configuration, whereas the addition of D-glucuronic acid and cellobiose $(1 \mathrm{mM})$ as tasteless sugars did not affect signal. Based on these results, our sweet sensors could differentiate a target sugar with excellent selectivity from the tasteless sugars.

The normalized sensitivity $\left(\Delta I / \Delta I_{\max }\right)$ of the DBT was demonstrated using various concentrations of the sweet tastants, as shown in Figure 6d. Increased sensitivity appeared when the DBT was treated with higher concentrations of natural (sucrose and fructose) and artificial (saccharin and aspartame) sweet tastants. However, no significant sensitivity changes were observed in the case of tasteless sweet tastants (Dglucuronic acid and cellobiose). Importantly, the DBT had a more sensitive reaction with the artificial tastants than the natural tastants, which is consistent with general human sensory responses. ${ }^{35}$ Furthermore, the normalized sensitivity showed lower threshold concentrations and wider detection ranges than those of nanovesicle-based bioelectronic tongues (NBTs). ${ }^{17}$ This result suggested that the artificial tastants elicited stronger stimulation than the natural tastants, and the DBT could mimic the sweet taste reception of a human with a very high sensitivity and is more qualified for quantitative analysis.

Dual Responses of the DBT for Umami and Sweet Tastants. In this study, our DBT platform delineated highly sensitive and selective responses for each umami and sweet tastant. Based on the outstanding performance, we used duplex sensor to simultaneously detect umami and sweet tastants. One channel was utilized as umami channel treated with umami taste receptor nanovesicles, and the other channel operated with sweet taste receptor nanovesicles. Figure 7a shows the dual responses in the DBT sensor upon the stimulation of tastants. The umami channel was stimulated only by the injection of MSG, whereas the sweet channel was selectively activated by the addition of sucrose and cyclamate. According to the selective interaction of each channel, the DBT system successfully discriminated specific tastants. Another dual observation was performed to verify the enhancing property of cyclamate for an umami channel (Figure 7b). The introduction of the cyclamate affected the current changes through the relation between the pre-existing MSG and the cyclamate, indicating that the cyclamate can act as an enhancer in the duplex system, as well as single-channel sensing system. Our DBT demonstrates human-like dual responses to umami and sweet tastants and has potential for applications such as bioelectronic tongues, which can mimic human sensation.

To validate the possibility of the DBT sensor for practical applications, responses for drink samples were observed using the duplex device. Commercial tomato juice and green tea were used for the drink samples (see the Materials and Methods section for the sampling method). Figure $7 \mathrm{c}$ depicts the result of dual responses for the tomato sample. Tomato contains much L-glutamate. ${ }^{40}$ The electrical current changes were detected in both the umami and sweet channels when the tomato juice solution was added. When the tomato juice solution additionally containing sucrose was subsequently added to the sensor, the current signal was further changed in the sweet channel. However, no signal change was detected in the umami channel. The response speed of the duplex sensor was slow in the case of the tomato sample. Presumably, the interaction between the nanovesicles and tastants could be interrupted by various types of nontarget molecules in the real drink sample. To further evaluate the sensing performance of a food sample, a green tea sample was used on the duplex system, as shown in Figure $7 \mathrm{~d}$. The selective responses from two channels occurred upon the exposure to the green tea sample. Green tea contains high level of amino acids including Lglutamate and is known to have umami taste. ${ }^{41,42}$ The addition of the green tea solution changed the current at only the umami channel. In contrast, the introduction of the solution containing sucrose altered the current signals of only the sweet channel. These results indicate that our DBT sensor has the ability to discriminate target tastants in real food samples and can be utilized in practical applications.

\section{CONCLUSIONS}

A human mimicking DBT was successfully demonstrated based on the heterodimeric human taste receptor nanovesicles immobilized on the duplex graphene electrodes. Each of the sensors showed high sensitivity and selectivity. It had the ability to detect the taste enhancing effect as in the human sensory system. Moreover, the duplex detections showed the humanlike responses to the umami and sweet tastants and ability to distinguish the target tastants in food samples. The DBT was used to monitor the interactions between the tastants and the umami taste receptors, exhibiting its practicality by mimicking human taste reception. The DBT platform overcomes the limitations of the previous artificial taste sensors such as low selectivity, low sensitivity, and impossibility to analyze unknown compounds. These limitations were an obstacle to mimic human taste reception. This sensor is expected to replace previous sensory evaluation strategies for the food and beverage industry and facilitate the functional study of dimeric GPCRs.

\section{MATERIALS AND METHODS}

Cell Culture and Construction of HEK-293 Cells Expressing Umami and Sweet Taste Receptors. Human embryonic kidney293 (HEK-293) cells were cultured in Dulbecco's modified Eagle medium (DMEM), supplemented with $10 \%$ fetal bovine serum and $1 \%$ penicillin-streptomycin (Gibco), at $37{ }^{\circ} \mathrm{C}$ under $5 \% \mathrm{CO}_{2}$. The cells were transfected with the mammalian expression vector pCMV6ENTRY containing human TAS1R3 using Lipofectamine3000 (Invitrogen) according to the manufacturer's instructions. After the transfection, cells were cultured for 1 day and transferred to the media containing G-418 $(1 \mathrm{mg} / \mathrm{mL})$ for selection. After culture for 1 week, G-418 resistant cell colonies were separately picked up and cultured in a fresh medium containing G-418. The stable expression of T1R3 on the cell membrane was confirmed by the Western blot analysis. The HEK-293 stable cell lines expressing T1R3 were transfected with pCMV6-ENTRY-hTAS1R1 and pCMV6-ENTRY-hTAS1R2 each by using Lipofectamine3000 (Invitrogen). After the transfection, the expression of T1R1 and T1R2 was confirmed by Western blot analysis.

Construction of Nanovesicles from HEK-293 Expressing Human Umami and Sweet Taste Receptor. HEK-293 cells expressing human umami and sweet taste receptors were suspended in serum free DMEM containing cytochalasin B $(20 \mu \mathrm{g} / \mathrm{mL}$, Sigma, USA) were incubated at $37^{\circ} \mathrm{C}$ with $300 \mathrm{rpm}$ agitation for $20 \mathrm{~min}$. Nanovesicles were separated from cells and cell debris by centrifugation (1000g, $5 \mathrm{~min}$ for cells; $2000 \mathrm{~g}, 20 \mathrm{~min}$ for cell debris) 
in an Eppendorf tube and collected by centrifugation at $12000 \mathrm{~g}$ for 30 min. The nanovesicles were finally resuspended in Dulbecco's phosphate buffered saline (DPBS, Gibco, USA). The produced nanovesicles were used immediately or stored at $-80{ }^{\circ} \mathrm{C}$ for subsequent experiments.

Immunoblot Analysis. HEK-293 cells of nanovesicles expressing umami and sweet taste receptors were lysed by sonication, and the membrane fractions were collected by centrifugation $(12000 \mathrm{~g}, 30$ $\mathrm{min})$. The pellet fractions were resuspended in PBS. Samples were mixed with $10 \%$ sodium dodecyl sulfate (SDS), $10 \% \beta$-mercaptonethanol, $0.3 \mathrm{M}$ Tris- $\mathrm{HCl}$ (pH 6.8), 0.05\% bromophenol blue, and 50\% glycerol and boiled for 5-10 min. The same amount of sample was loaded onto 7.5\% PAGE gels (Laemmli) and electrophoresed at $80 \mathrm{~V}$. The proteins in the gel were transferred to nitrocellulose membrane under a constant current setting of $0.15 \mathrm{~A}$ for $60 \mathrm{~min}$. The membrane was incubated in blocking solution ( 5 wt \% skim milk in PBS containing 0.1 vol \% Tween-20) at $25{ }^{\circ} \mathrm{C}$ for $1 \mathrm{~h}$. The blocked membrane was incubated in the solution of primary antibody (antiFLAG antibody for T1R3 stable cell line, anti-T1R1 antibody for transient expression of T1R1 in stable cell line with T1R3, anti-T1R2 antibody for transient expression of T1R2 in stable cell line with T1R3 from rabbit, 1:500 dilution with $1 \%$ skim milk in TBS-T) at $4{ }^{\circ} \mathrm{C}$ for overnight. The antibody-treated membrane was washed with TBS-T several times and incubated with HRP-conjugated anti-rabbit antibody ( $1: 1000$ dilution with $5 \%$ skim milk in TBS-T) at $25{ }^{\circ} \mathrm{C}$ for $1 \mathrm{~h}$. After membranes were washed several times with TBS-T, Western blotting was performed using an enhanced chemiluminescence detection kit (Amersham Pharmacia Biotech, UK).

Intracellular Calcium Assay. For calcium signaling analysis, HEK-293 cells expressing umami and sweet taste receptors were cultured for more than 2 days and incubated in Ringer's solution (140 $\mathrm{mM} \mathrm{NaCl}, 1 \mathrm{mM} \mathrm{MgCl}, 1.8 \mathrm{mM} \mathrm{CaCl}_{2}, 5 \mathrm{mM} \mathrm{KCl}, 5 \mathrm{mM}$ glucose, and $10 \mathrm{mM}$ 4-(2-hydroxyethyl)-1-piperazineethanesulfonic acid (HEPES), $\mathrm{pH}$ 7.4) containing $10 \mathrm{mM}$ Fura-2/AM (Invitrogen, USA) at $37{ }^{\circ} \mathrm{C}$ for $30 \mathrm{~min}$. After incubation, the cells were washed several times with the same buffer solution without Fura-2/AM, and the cleavage of the AM ester group was followed by incubation for $1 \mathrm{~h}$ at $37^{\circ} \mathrm{C}$. The fluorescence signal upon the addition of tastants and ATP $(100 \mu \mathrm{M})$ was measured at $510 \mathrm{~nm}$ by dual excitation at 340 and $380 \mathrm{~nm}$, using a spectrofluorophotometer (Tecan). For calcium analysis of nanovesicles, nanovesicles containing umami and sweet taste receptors were generated from Fura2-AM loaded cells, according to the process described above. Then, the nanovesicles were immobilized on poly(D-lysine)-treated 96-wells plates and incubated at $37{ }^{\circ} \mathrm{C}$ for $2 \mathrm{~h}$. The calcium signals upon the addition of tastants were measured by the same processor of calcium signaling analysis.

Fabrication of Duplex Graphene Electrodes. Single layer graphene was first fabricated by the chemical vapor deposition (CVD) process as reported previously. ${ }^{28}$ Subsequently, the graphene was transferred on a silicon substrate by the dry-transfer method. Duplex graphene electrodes (DGEs) were prepared using a typical photolithography process as follows. The graphene substrate was coated with a GXR601 photoresist by the spin-coating method, and square patterns were created using photolithography (Karl-Süss, MA-6). Unpatterned region of the graphene substrate was etched by the reactive ion etching (RIE) process (Oxford Instruments), and then an AZ300 remover was used to remove the patterned photoresist. This graphene patterns were utilized for the channel region of DGEs. Afterward, second photolithography process was conducted to fabricate the patterns of contact electrode. The contact electrodes were deposited by the thermal evaporation of $\mathrm{Cr} / \mathrm{Au}(10 \mathrm{nM} / 40 \mathrm{nM})$ and lift-off method.

Immobilization of Taste Nanovesicles on the Surface of DGEs. The prepared DGEs were exposed to $1 \mathrm{mM}$ 1-pyrenebutanoic acid $N$-hydroxysuccinimidyl ester (PSE) solution in methanol for 30 min at room temperature, and then the DGEs were rinsed with pure methanol and modified through $\pi$-stacking between the pyrene groups of the PSE and the surface of the graphene. To immobilize taste receptor nanovesicles on the surface of the DGEs, the modified DGEs were dipped in nanovesicle solution for $3 \mathrm{~h}$. The succinimidyl groups of the PSE interacted with the proteins of the nanovesicles, forming peptide bonds. As a result, taste nanovesicles were successfully immobilized on the surface of the DGEs.

Sensing Measurements. A Keithley 2612A semiconductor analyzer, a probe station (MS TECH, model 4000), and a multichannel system (ONTEST Co.) were used to measure the electrical performance of the DBTs. DPBS containing $2 \mathrm{mM} \mathrm{CaCl}_{2}$ at $\mathrm{pH} 7.4$ was utilized as the electrolyte. Then, the DPBS was placed in a glass chamber (200 $\mu \mathrm{L}$ volume). Electrical current signal was detected and normalized as $\Delta I / I_{0}=\left(I-I_{0}\right) / I_{0}$, where $I$ is the detected real-time current and $I_{0}$ is the initial current.

Preparation of Tastants. Umami and sweet tastants in this study were purchased from Sigma-Aldrich. Tastants were solubilized in Dulbecco's phosphate buffered saline (DPBS, Gibco, USA) at various concentrations. The artificial seasoning 1 , containing salt, sugars (sugar, lactose), flavor raw material (dried bonito powder and you extract), yeast extract, wheat protein fermented seasoning, and yeast extract fermented seasoning/seasoning (such as amino acids), and artificial seasoning 2, containing 97.3\% MSG, 1.35\% IMP, and 1.35\% GMP, were purchased commercially and solubilized in pure water at various concentrations. Tomato juice (containing tomato juice, purified water, liquid exaggeration, citric acid, synthetic flavors, cellulose gum, rock pigments, and vitamin C) was also purchased, and sucrose was added to it at $100 \mu \mathrm{M}$. For green tea, a green tea bag was placed in $100 \mathrm{~mL}$ of water at $90{ }^{\circ} \mathrm{C}$ for $5 \mathrm{~min}$, and a teaspoon of sucrose was added $(1 \mathrm{mM})$.

\section{ASSOCIATED CONTENT}

\section{S Supporting Information}

The Supporting Information is available free of charge on the ACS Publications website at DOI: 10.1021/acsnano.6b02547.

Additional information about the calcium signal analysis of monomeric receptor expressing cells, the photograph of DBT circuit and the HR-TEM image of single layer graphene (PDF)

\section{AUTHOR INFORMATION}

\section{Corresponding Authors}

*E-mail: thpark@snu.ac.kr.

*E-mail: jsjang@plaza.snu.ac.kr.

\section{Author Contributions}

II S.R.A. and J.H.A. contributed equally to this study.

\section{Notes}

The authors declare no competing financial interest.

\section{ACKNOWLEDGMENTS}

This research was supported by the National Research Foundation funded by the Korean government (MSIP, No. 2015065103, and MEST, No. 2011-0017125).

\section{REFERENCES}

(1) Palmer, R. K. The Pharmacology and Signaling of Bitter, Sweet, and Umami Taste Sensing. Mol. Interventions 2007, 7, 87-98.

(2) Adler, E.; Hoon, M. A.; Mueller, K. L.; Chandrashekar, J.; Ryba, N. J. P.; Zuker, C. S. A Novel Family of Mammalian Taste Receptors. Cell 2000, 100, 693-702.

(3) Nelson, G.; Chandrashekar, J.; Hoon, M. A.; Feng, L.; Zhao, G.; Ryba, N. J.; Zuker, C. S. An Amino-Acid Taste Receptor. Nature 2002, 416, 199-202.

(4) Li, X.; Staszewski, L.; Xu, H.; Durick, K.; Zoller, M.; Adler, E. Human Receptors for Sweet and Umami Taste. Proc. Natl. Acad. Sci. U. S. A. 2002, 99, 4692-4696.

(5) Nelson, G.; Hoon, M. A.; Chandrashekar, J.; Zhang, Y.; Ryba, N. J.; Zuker, C. S. Mammalian Sweet Taste Receptors. Cell 2001, 106, 381-390. 
(6) Hui, G. H.; Mi, S. S.; Deng, S. P. Sweet and Bitter Tastants Specific Detection by the Taste Cell-Based Sensor. Biosens. Bioelectron. 2012, 35, 429-438.

(7) Zhao, G. Q.; Zhang, Y.; Hoon, M. A.; Chandrashekar, J.; Erlenbach, I.; Ryba, N. J.; Zuker, C. S. The Receptors for Mammalian Sweet and Umami Taste. Cell 2003, 115, 255-266.

(8) Harada, T.; Uchida, T.; Yoshida, M.; Kobayashi, Y.; Narazaki, R.; Ohwaki, T. A New Method for Evaluating the Bitterness of Medicines in Development Using a Taste Sensor and a Disintegration Testing Apparatus. Chem. Pharm. Bull. 2010, 58, 1009-1014.

(9) Riul, A., Jr; Malmegrim, R.; Fonseca, F.; Mattoso, L. An Artificial Taste Sensor Based on Conducting Polymers. Biosens. Bioelectron. 2003, 18, 1365-1369.

(10) Yu, N.; Atienza, J. M.; Bernard, J.; Blanc, S.; Zhu, J.; Wang, X.; Xu, X.; Abassi, Y. A. Real-Time Monitoring of Morphological Changes in Living Cells by Electronic Cell Sensor Arrays: An Approach To Study G Protein-Coupled Receptors. Anal. Chem. 2006, 78, 35-43.

(11) Toko, K. Taste Sensor. Sens. Actuators, B 2000, 64, 205-215.

(12) Tahara, Y.; Maehara, Y.; Ke, J.; Ikeda, A.; Toko, K. Development of a Multichannel Taste Sensor Chip for a Portable Taste Sensor. 2012 IEEE Sensors; IEEE: Piscataway, NJ, 2012; pp 1-4.

(13) Halder, A.; Mahato, M.; Sinha, T.; Adhikari, B.; Mukherjee, S.; Bhattacharyya, N. Polymer Membrane Electrode Based Potentiometric Taste Sensor: A New Sensor to Distinguish Five Basic Tastes. 2012 Sixth International Conference on Sensing Technology (ICST); IEEE: Piscataway, NJ, 2012; pp 785-789.

(14) Hayashi, K.; Yamanaka, M.; Toko, K.; Yamafuji, K. Multichannel Taste Sensor Using Lipid-Membranes. Sens. Actuators, B 1990, 2, 205213.

(15) Morini, G.; Bassoli, A.; Temussi, P. A. From Small Sweeteners to Sweet Proteins: Anatomy of the Binding Sites of the Human T1R2 T1R3 Receptor. J. Med. Chem. 2005, 48, 5520-5529.

(16) Song, H. S.; Kwon, O. S.; Lee, S. H.; Park, S. J.; Kim, U.-K.; Jang, J.; Park, T. H. Human Taste Receptor-Functionalized Field Effect Transistor as a Human-Like Nanobioelectronic Tongue. Nano Lett. 2013, 13, 172-178.

(17) Song, H. S.; Jin, H. J.; Ahn, S. R.; Kim, D.; Lee, S. H.; Kim, U. K.; Simons, C. T.; Hong, S.; Park, T. H. Bioelectronic Tongue Using Heterodimeric Human Taste Receptor for the Discrimination of Sweeteners with Human-like Performance. ACS Nano 2014, 8, 97819789.

(18) Bockaert, J.; Pin, J. P. Molecular Tinkering of G ProteinCoupled Receptors: An Evolutionary Success. EMBO J. 1999, 18, 1723-1729.

(19) Thomsen, W.; Frazer, J.; Unett, D. Functional Assays for Screening GPCR Targets. Curr. Opin. Biotechnol. 2005, 16, 655-665.

(20) Xu, H.; Staszewski, L.; Tang, H.; Adler, E.; Zoller, M.; Li, X. Different Functional Roles of T1R Subunits in the Heteromeric Taste Receptors. Proc. Natl. Acad. Sci. U. S. A. 2004, 101, 14258-14263.

(21) Lindemann, B. Taste Reception. Physiol. Rev. 1996, 76, 718766.

(22) Chandrashekar, J.; Hoon, M. A.; Ryba, N. J.; Zuker, C. S. The Receptors and Cells for Mammalian Taste. Nature 2006, 444, 288294.

(23) Dong, H.; Dunn, J.; Lytton, J. Stoichiometry of the Cardiac $\mathrm{Na}^{+} / \mathrm{Ca}^{2+}$ Exchanger NCX1.1 Measured in Transfected HEK Cells. Biophys. J. 2002, 82, 1943-1952.

(24) Liu, M.; Chen, T. Y.; Ahamed, B.; Li, J.; Yau, K. W. CalciumCalmodulin Modulation of the Olfactory Cyclic Nucleotide-Gated Cation Channel. Science 1994, 266, 1348-1354.

(25) Schedin, F.; Geim, A. K.; Morozov, S. V.; Hill, E. W.; Blake, P.; Katsnelson, M. I.; Novoselov, K. S. Detection of Individual Gas Molecules Adsorbed on Graphene. Nat. Mater. 2007, 6, 652-655.

(26) Li, X.; Zhu, Y.; Cai, W.; Borysiak, M.; Han, B.; Chen, D.; Piner, R. D.; Colombo, L.; Ruoff, R. S. Transfer of Large-Area Graphene Films for High-Performance Transparent Conductive Electrodes. Nano Lett. 2009, 9, 4359-4363.
(27) Park, S. J.; Kwon, O. S.; Lee, S. H.; Song, H. S.; Park, T. H.; Jang, J. Ultrasensitive Flexible Graphene Based Field-Effect Transistor (FET)-Type Bioelectronic Nose. Nano Lett. 2012, 12, 5082-5090.

(28) An, J. H.; Park, S. J.; Kwon, O. S.; Bae, J.; Jang, J. HighPerformance Flexible Graphene Aptasensor for Mercury Detection in Mussels. ACS Nano 2013, 7, 10563-10571.

(29) Kwon, O. S.; Song, H. S.; Park, S. J.; Lee, S. H.; An, J. H.; Park, J. W.; Yang, H.; Yoon, H.; Bae, J.; Park, T. H.; Jang, J. An Ultrasensitive, Selective, Multiplexed Superbioelectronic Nose That Mimics the Human Sense of Smell. Nano Lett. 2015, 15, 6559-6567.

(30) Ebrish, M. A.; Olson, E. J.; Koester, S. J. Effect of Noncovalent Basal Plane Functionalization on the Quantum Capacitance in Graphene. ACS Appl. Mater. Interfaces 2014, 6, 10296-10303.

(31) Feifel, S. C.; Stieger, K. R.; Lokstein, H.; Lux, H.; Lisdat, F. High Photocurrent Generation by Photosystem I on Artificial Interfaces Composed of $\pi$-System-Modified Graphene. J. Mater. Chem. A 2015, 3, 12188-12196.

(32) Graf, D.; Molitor, F.; Ensslin, K.; Stampfer, C.; Jungen, A.; Hierold, C.; Wirtz, L. Spatially Resolved Raman Spectroscopy of Single- and Few-Layer Graphene. Nano Lett. 2007, 7, 238-242.

(33) Kwon, O. S.; Lee, S. H.; Park, S. J.; An, J. H.; Song, H. S.; Kim, T.; Oh, J. H.; Bae, J.; Yoon, H.; Park, T. H.; Jang, J. Large-Scale Graphene Micropattern Nano-biohybrids: High-Performance Transducers for FET-Type Flexible Fluidic HIV Immunoassays. Adv. Mater. 2013, 25, 4177-4185.

(34) Xu, H.; Staszewski, L.; Tang, H.; Adler, E.; Zoller, M.; Li, X. Different Functional Roles of T1R Subunits in the Heteromeric Taste Receptors. Proc. Natl. Acad. Sci. U. S. A. 2004, 101, 14258-14263.

(35) Yamaguchi, S. Basic Properties of Umami and Its Effects on Food Flavor. Food Rev. Int. 1998, 14, 139-176.

(36) Osawa, Y. Glutamate Perception, Soup Stock, and the Concept of Umami: The Ethnography, Food Ecology, and History of Dashi in Japan. Ecol. Food Nutr. 2012, 51, 329-345.

(37) Yamaguchi, S.; Ninomiya, K. Umami and Food Palatability. J. Nutr. 2000, 130, 921S-926S.

(38) Rhodes, J.; Titherley, A. C.; Norman, J. A.; Wood, R.; Lord, D. W. A Survey of the Monosodium Glutamate Content of Foods and an Estimation of the Dietary Intake of Monosodium Glutamate. Food Addit. Contam. 1991, 8, 663-672.

(39) Loliger, J. Function and Importance of Glutamate for Savory Foods. J. Nutr. 2000, 130, 915s-920s.

(40) Ninomiya, K. Umami: A Universal Taste. Food Rev. Int. 2002, $18,23-38$.

(41) Hayashi, N.; Chen, R.; Ikezaki, H.; Ujihara, T. Evaluation of the Umami Taste Intensity of Green Tea by a Taste Sensor. J. Agric. Food Chem. 2008, 56, 7384-7387.

(42) Kaneko, S.; Kumazawa, K.; Masuda, H.; Henze, A.; Hofmann, T. Molecular and Sensory Studies on the Umami Taste of Japanese Green Tea. J. Agric. Food Chem. 2006, 54, 2688-2694. 